\title{
Exponentially stable equilibria to an indefinite nonlinear Neumann problem in smooth domains
}

\author{
Gustavo Ferron Madeira and Arnaldo Simal do Nascimento
}

\begin{abstract}
We prove existence of two nonconstant exponentially stable equilibria to the heat equation supplied with a nonlinear Neumann boundary condition in any smooth $n$-dimensional domain $(n \geq 2)$, independently of its geometry. The Neumann boundary condition reflects the fact that the flux on the boundary is proportional to the product of a prescribed bistable function of the density or concentration with an indefinite weight. Such solutions are obtained via variational methods, by minimizing the corresponding energy functional on suitable invariant sets to the semiflow generated by the parabolic problem. But this is possible only if the parameter in the boundary condition is sufficiently large, otherwise we prove using the Implicit Function Theorem the uniqueness of constant equilibrium solutions. The same theorem allows us to derive isolation and smooth dependence on the parameter for nonconstant exponentially stable equilibria found.
\end{abstract}

Mathematical Subject Classification (2000). 37L15; 35K57; 35K55; 35J60; 35 B33.

Keywords. Nonconstant stable equilibria, Nonlinear boundary condition, Indefinite weight, Nonlinear heat equation, Bistable function.

\section{Introduction}

An important question in problems modeling time-dependent phenomena is stability of its stationary solutions (also called equilibrium solutions or equilibria, for short). For instance, it is well known that for some semilinear parabolic equations with gradient-like structure, equilibrium solutions play a fundamental role since understanding the long term dynamics depends heavily on its existence and stability. For further discussions and applications on this subject, see for example $[3,5,13,15]$ and the references therein.

The research of G. F. Madeira was partially supported by Capes/Brazil. 
Regarding questions related to the problem of existence of nonconstant stable equilibria to parabolic problems under linear or nonlinear boundary conditions, the geometry of the domain plays a fundamental role in answering many of them. In fact, it can be responsible for existence or nonexistence results according to suitable hypothesis, as will be seen below. In this work we are concerned with the same problem in the nonlinear boundary condition case, but we can give an answer for arbitrary smooth domains. Namely, we prove the existence of two nonconstant exponentially stable equilibria to a heat equation under a nonlinear Neumann boundary condition, independently of domain geometry. To be more precise, we consider the problem

$$
\left\{\begin{array}{lll}
\partial_{t} u=\Delta u & \text { in } & \Omega \times \mathbb{R}^{+} \\
\frac{\partial u}{\partial \nu}=\varepsilon \eta(x) g(u) & \text { on } & \partial \Omega \times \mathbb{R}^{+}
\end{array}\right.
$$

where $\Omega \subset \mathbb{R}^{n}(n \geq 2)$ is a bounded smooth domain, $\nu$ the outward pointing normal vector, $\varepsilon>0$ a parameter and $g(u)=u-u^{3}$ is the prototype balanced bistable function.

We suppose that $\eta \in C^{1, \gamma}(\partial \Omega)$, for some $0<\gamma<1$, satisfies

(H) $\quad \eta$ is an indefinite weight and $\int_{\partial \Omega} \eta(x) d \mathcal{H}^{n-1} \neq 0$

where $\mathcal{H}^{n-1}$ denotes the $(n-1)$-dimensional Hausdorff measure and an indefinite weight means a function which assume both positive and negative values.

Typically (1) models a diffusion problem with the flux on the boundary being proportional to the product of a function of the density or concentration with a prescribed function on the boundary which depends on the spatial variable only. See [15], for instance, for the derivation of some specific models with nonlinear boundary conditions.

Our main goal in this paper is to prove existence of two nonconstant exponentially stable equilibria to (1) - stability in Lyapunov sense- one of them positive and the other one negative, if $\varepsilon>0$ is sufficiently large in any $n$-dimensional smooth domain $(n \geq 2)$. This condition on the parameter is actually necessary. Indeed, there is no nonconstant stable equilibria to (1) when the parameter is small because we prove that there are no equilibria to (1) besides the constant ones if $\varepsilon>0$ is small enough. It is also proved that nonconstant stable equilibria got are isolated and depend smoothly on the parameter. We consider the function $g(u)=u-u^{3}$ just for simplicity but as long as our results are concerned it could have been replaced with any smooth bistable type odd functions such that there are real numbers $\alpha$ and $\beta(\alpha<\beta)$ with $g(\alpha)=g(\beta)=0, g^{\prime}(\alpha)=g^{\prime}(\beta)<0$.

Let us now put our work under the perspective of some known results. When $\eta \equiv$ const, that is, when there is no explicit spatial dependence in the boundary condition, the geometry of the domain determines the existence of nonconstant stable equilibrium solutions to (1). Indeed if $\Omega=B_{R}(0)$ (the $n$-dimensional ball with radius $R$ and centered at the origin) then there is no nonconstant stable equilibrium solution to (1) whereas such solutions can be created for dumbbell type, thus non-convex, domains. See $[10,11]$. 
When $n=2, \eta \equiv 1$ and $g(x, u)=u(1-u)(c(x)-u)$, where $0<c(x)<1$, existence of nonconstant stable equilibrium solutions to (1) was proved in [8] for smooth planar domains. Our work corroborates the assertion that explicit spatial dependence on boundary condition for reaction-diffusion equations can be a mechanism to create nonconstant stable equilibrium solutions. We emphasize that, under our hypotheses, problem (1) exemplifies this property can holds true for arbitrary smooth domains $\Omega \subset \mathbb{R}^{n}, n \geq 2$.

The procedure we use herein is the following: first, we minimize the energy functional corresponding to the stationary problem associated to (1) in suitable invariant sets of the semiflow generated by (1). The equilibrium problem corresponding to (1) and the invariant sets are given in Sect. 2. Such a minimization process yields two relative minimizers which are proved to be (weak) equilibrium solutions. This can be done by choosing suitable directions for derivative exploring the geometry of the invariant sets. The fact that the boundary weight changes sign implies the existence of a positive solution to an eigenvalue problem which is used to assure the minimizers are nonconstant. But a necessary condition for this scheme to be successful is the parameter $\varepsilon>0$ being not small. In fact, this is a consequence of the uniqueness result proved in Sect. 3 with the help of the Implicit Function Theorem, namely, all equilibrium solutions to (1) are constant if the parameter is small. It should be remarked that such result is valid for more general functions $g$.

Finally, in Sect. 5, we prove the exponential stability of nonconstant equilibria got before. This is carried out by proving first that zero is not in the spectrum of the corresponding linearized eigenvalue problem to (1). Then by remarking that relative minimizers of the energy above mentioned in fact locally minimize it in a suitable topology, we can infer the desired stability property via linearized stability principle. Using again the Implicit Function Theorem, the paper is finished by establishing isolation and smooth dependence on the parameter for nonconstant stable equilibria previously found.

\section{Invariant sets}

Problem (1) generates a semiflow in $H^{1}(\Omega)$ for a large class of boundary nonlinearities $g$, see $[3,5,7]$ for instance. Moreover, the semiflow is in fact a gradient system and, therefore, the orbits approach the equilibrium solutions as $t$ goes to infinity.

Consider the closed subsets of $H^{1}(\Omega)$ given by

$$
\begin{aligned}
\mathfrak{M} & \doteq\left\{v \in H^{1}(\Omega):-1 \leq v(x) \leq 1 \quad \text { a.e. } \quad x \in \Omega\right\} \\
\mathfrak{M}^{-} \doteq\left\{v \in H^{1}(\Omega):-1 \leq v(x) \leq 0 \quad\right. \text { a.e. } & x \in \Omega\} \\
\mathfrak{M}^{+} \doteq\left\{v \in H^{1}(\Omega): \quad 0 \leq v(x) \leq 1 \quad\right. \text { a.e. } & x \in \Omega\} .
\end{aligned}
$$

These sets will be important in treating (1) because of their invariance under the semiflow generated by $(1)$ when $H^{1}(\Omega)$ is the phase-space, according to 
Theorem 2.1. The sets in (2) are positively invariant to the semiflow generated by (1) in $H^{1}(\Omega)$.

Proof. We will only sketch the proof for $\mathfrak{M}$. For more details, see similar argumentation in [14]. Let $u_{0} \in \mathfrak{M}$ be an initial datum and let $u$ be the (classical) solution to $(1)$ such that $u\left(\cdot, 0 ; u_{0}\right)=u_{0}$. Taking a sequence $\left\{\zeta_{k}\right\} \subset C^{\infty}(\bar{\Omega})$ such that $\zeta_{k}>-1$ in $\bar{\Omega}$ converging to $u_{0}$ in $H^{1}(\Omega)$ as $k \rightarrow \infty$ (cf. Remark 2.1), by continuity we have $T_{k}>0$ such that

$$
u\left(x, t ; \zeta_{k}\right)>-1 \quad \forall(x, t) \in \bar{\Omega} \times\left[0, T_{k}\right) .
$$

Using suitably the maximum principle, one can infer that $T_{k}=\infty$ for all $k$ and since

$$
\left\|u\left(\cdot, t ; \zeta_{k}\right)-u\left(\cdot, t ; u_{0}\right)\right\|_{H^{1}(\Omega)} \stackrel{k \rightarrow \infty}{\longrightarrow} 0
$$

for each $t \geq 0$, we have

$$
u\left(x, t ; u_{0}\right) \geq-1 \quad \text { a.e. } x \in \Omega, \quad \forall t \geq 0 .
$$

With an analogous argument one can conclude that

$$
u\left(x, t ; u_{0}\right) \leq 1 \quad \text { a.e. } x \in \Omega, \quad \forall t \geq 0,
$$

and thus the invariance of $\mathfrak{M}$.

Remark 2.1. In proving Theorem 2.1 it was used the fact that one can approximate in the $H^{1}(\Omega)$-norm an initial datum $u_{0} \in \mathfrak{M}$ by a sequence $\left\{\zeta_{k}\right\} \subset C^{\infty}(\bar{\Omega})$ such that $\zeta_{k}>-1$ in $\bar{\Omega}$. This follows from the fact that an almost everywhere nonnegative function of $W_{p}^{m}(\Omega)$ can be approximated in the $W_{p}^{m}(\Omega)$-norm by a sequence of nonnegative functions in $C^{\infty}(\bar{\Omega})$ for all $m \geq 1$ and $1 \leq p<\infty$. This in turn can be proved using mollification and partitions of unity.

Therefore, by Theorem 2.1 the map

$$
\mathfrak{M} \ni u_{0} \longmapsto u\left(\cdot, t ; u_{0}\right) \in \mathfrak{M}, \quad t \geq 0
$$

defined through solutions to (1) is a semiflow, with a Lyapunov function $\mathcal{E}_{\varepsilon}$ : $H^{1}(\Omega) \longrightarrow \mathbb{R}$, given by

$$
\mathcal{E}_{\varepsilon}(u)=\frac{1}{2} \int_{\Omega}|\nabla u|^{2} d x-\varepsilon \int_{\partial \Omega} \eta(x)\left[\frac{u^{2}}{2}-\frac{u^{4}}{4}\right] d \mathcal{H}^{n-1}
$$

for all $\varepsilon>0$. Thus, with respect to the dynamical system above, the equilibrium solutions to (1) are the solutions to the elliptic problem

$$
\begin{cases}\Delta u=0 & \text { in } \quad \Omega \\ \frac{\partial u}{\partial \nu}=\varepsilon \eta(x) g(u) & \text { on } \quad \partial \Omega\end{cases}
$$

which belong to $\mathfrak{M}$, and the constant equilibrium solutions to (1) are $u \equiv$ $-1, u \equiv 0$ and $u \equiv 1$. The solutions we are looking for, namely, nonconstant equilibria to (1), will be found in the invariant sets $\mathfrak{M}^{-}$and $\mathfrak{M}^{+}$. But first it will be proved that this is possible only for large values of the parameter. 


\section{Uniqueness of constant equilibria for $\varepsilon>0$ small}

We shall prove in this section a theorem about uniqueness of the constant equilibrium solutions to (1) valid for all $\varepsilon>0$ sufficiently small. Such result holds true for nonlinearities in a wider class than of bistable type. First of all, carrying out an idea in [9] used to treat an elliptic problem under homogeneous Neumann boundary condition, we will derive a fundamental lemma based on the Implicit Function Theorem in order to get local uniqueness of the constant equilibrium solutions to (1). Then the main result will be derived by a contradiction argument through suitable estimates.

Lemma 3.1. Let $\bar{u}$ be a constant equilibrium solution to (1). Then, there exists a neighborhood of $\bar{u}$ in $W_{p}^{2}(\Omega), p>n$, on which $\bar{u}$ is the only solution to (4) for sufficiently small $\varepsilon>0$.

Proof. Consider the closed subspace of $L^{p}(\Omega) \times W_{p}^{1-1 / p}(\partial \Omega), p>n$,

$$
\mathfrak{U} \doteq\left\{(\phi, \psi) \in L^{p}(\Omega) \times W_{p}^{1-1 / p}(\partial \Omega): \int_{\Omega} \phi d x=\int_{\partial \Omega} \psi d \mathcal{H}^{n-1}\right\}
$$

and define $\Phi: \mathbb{R}^{+} \times W_{p}^{2}(\Omega) \longrightarrow \mathfrak{U} \times \mathbb{R}$ by

$$
\Phi(\varepsilon, u)=\left(\begin{array}{l}
\Delta u \\
\partial u / \partial \nu-\varepsilon \eta(\cdot) g(u)+\varepsilon f_{\partial \Omega} \eta(x) g(u) d \mathcal{H}^{n-1} \\
f_{\partial \Omega} \eta(x) g(u) d \mathcal{H}^{n-1}
\end{array}\right)
$$

where $f_{\partial \Omega}$ denotes the average over $\partial \Omega$. It follows that $\Phi$ is well defined and $u \in W_{p}^{2}(\Omega)$ is a solution to (4) if, and only if, $\Phi(\varepsilon, u)=0$. Note also that $\Phi(0, \bar{u})=0$ and every point of the curve

$$
\{(\varepsilon, \bar{u}): \varepsilon>0\} \subset \mathbb{R}^{+} \times W_{p}^{2}(\Omega)
$$

is a zero of $\Phi$. In order to apply the Implicit Function Theorem, we will follow two steps.

Step 1. $\Phi: \mathbb{R}^{+} \times W_{p}^{2}(\Omega) \longrightarrow \mathfrak{U} \times \mathbb{R}$ is smooth in Fréchet sense.

Note initially that by the chain rule, the relation $g\left(\left.u\right|_{\partial \Omega}\right)=\left.g(u)\right|_{\partial \Omega}$, which is valid for smooth functions $u$, and the fact that $\eta(\cdot)$ has a smooth extension to $\bar{\Omega}$, the function

$$
W_{p}^{2}(\Omega) \ni u \longmapsto \varepsilon \eta(\cdot) g(u) \in W_{p}^{1-1 / p}(\partial \Omega) \quad(p>n)
$$

is well defined and differentiable in Fréchet sense. Now, we shall only check that $\Phi$ is of class $C^{1}$ since this case contains the relevant arguments to infer that $\Phi$ is smooth. 
The Gateaux derivative with respect to the second argument of $\Phi$ at $(\varepsilon, u) \in \mathbb{R}^{+} \times W_{p}^{2}(\Omega)$ is given by

$$
D_{u} \Phi(\varepsilon, u) \cdot v=\left(\begin{array}{l}
\Delta v \\
\partial v / \partial \nu-\varepsilon \eta(\cdot) g^{\prime}(u) v+\varepsilon f_{\partial \Omega} \eta(x) g^{\prime}(u) v d \mathcal{H}^{n-1} \\
f_{\partial \Omega} \eta(x) g^{\prime}(u) v d \mathcal{H}^{n-1}
\end{array}\right)
$$

for all $v \in W_{p}^{2}(\Omega)$. To verify the continuity of $D_{u} \Phi$, take a sequence $\left\{\left(\varepsilon_{i}, u_{i}\right)\right\}$ converging to $(\varepsilon, u)$ in $\mathbb{R}^{+} \times W_{p}^{2}(\Omega)$ as $i \rightarrow \infty$. We need to prove that

$$
\vartheta_{i}:=\sup \left\{\left\|D_{u} \Phi\left(\varepsilon_{i}, u_{i}\right) \cdot v-D_{u} \Phi(\varepsilon, u) \cdot v\right\|_{\mathfrak{U} \times \mathbb{R}}:\|v\|_{W_{p}^{2}(\Omega)} \leq 1\right\} \stackrel{i \rightarrow \infty}{\longrightarrow} 0 .
$$

For all $v \in W_{p}^{2}(\Omega)$ with $\|v\|_{W_{p}^{2}(\Omega)} \leq 1$, we have

$$
\begin{array}{rl}
\left\|D_{u} \Phi\left(\varepsilon_{i}, u_{i}\right) \cdot v-D_{u} \Phi(\varepsilon, u) \cdot v\right\|_{\mathfrak{U} \times \mathbb{R}} \\
\leq C & C\left[\left\|\eta(\cdot)\left(\varepsilon g^{\prime}(u)-\varepsilon_{i} g^{\prime}\left(u_{i}\right)\right) v\right\|_{W_{p}^{1-1 / p}(\partial \Omega)}+\left\|\eta(\cdot)\left(\varepsilon_{i} g^{\prime}\left(u_{i}\right)-\varepsilon g^{\prime}(u)\right) v\right\|_{L^{1}(\partial \Omega)}\right. \\
& \left.+\left\|\eta(\cdot)\left(g^{\prime}\left(u_{i}\right)-g^{\prime}(u)\right) v\right\|_{L^{1}(\partial \Omega)}\right] \\
\leq & C\left[\left\|\left(\varepsilon g^{\prime}(u)-\varepsilon_{i} g^{\prime}\left(u_{i}\right)\right)\right\|_{W_{p}^{1}(\Omega)}\|v\|_{W_{p}^{1}(\Omega)}+\left\|\varepsilon_{i} g^{\prime}\left(u_{i}\right)-\varepsilon g^{\prime}(u)\right\|_{L^{p^{\prime}}(\partial \Omega)}\|v\|_{W_{p}^{2}(\Omega)}\right. \\
& \left.+\left\|g^{\prime}\left(u_{i}\right)-g^{\prime}(u)\right\|_{L^{p^{\prime}(\partial \Omega)}}\|v\|_{W_{p}^{2}(\Omega)}\right] \\
\leq & C\left[\left\|\varepsilon g^{\prime}(u)-\varepsilon_{i} g^{\prime}\left(u_{i}\right)\right\|_{W_{p}^{1}(\Omega)}+\left\|\varepsilon_{i} g^{\prime}\left(u_{i}\right)-\varepsilon g^{\prime}(u)\right\|_{L^{p^{\prime}}(\partial \Omega)}\right. \\
\left.\quad+\left\|g^{\prime}\left(u_{i}\right)-g^{\prime}(u)\right\|_{L^{p^{\prime}}(\partial \Omega)}\right]
\end{array}
$$

where the constants in the previous estimates, which do not depend on $v$, were denoted by $C>0,1 / p+1 / p^{\prime}=1$, and we used the Trace Theorem and the fact that $W_{p}^{k}(\Omega)(k=1,2)$ is a Banach Algebra in our hypotheses (see [1]). Then

$$
\begin{aligned}
\vartheta_{i} \leq & C\left[\left\|\varepsilon g^{\prime}(u)-\varepsilon_{i} g^{\prime}\left(u_{i}\right)\right\|_{W_{p}^{1}(\Omega)}+\left\|\varepsilon_{i} g^{\prime}\left(u_{i}\right)-\varepsilon g^{\prime}(u)\right\|_{L^{p^{\prime}}(\partial \Omega)}\right. \\
& \left.+\left\|g^{\prime}\left(u_{i}\right)-g^{\prime}(u)\right\|_{L^{p^{\prime}}(\partial \Omega)}\right] .
\end{aligned}
$$

The $\mathbb{R}^{+} \times W_{p}^{2}(\Omega)$-convergence of $\left\{\left(\varepsilon_{i}, u_{i}\right)\right\}$ to $(\varepsilon, u)$ imply the convergence to zero of the right hand-side of the last inequality since

$$
u_{i} \stackrel{i \rightarrow \infty}{\longrightarrow} u, \quad \nabla u_{i} \stackrel{i \rightarrow \infty}{\longrightarrow} \nabla u \quad \text { and }\left.\left.\quad u_{i}\right|_{\partial \Omega} \stackrel{i \rightarrow \infty}{\longrightarrow} u\right|_{\partial \Omega}
$$

uniformly because $p>n$. Thus, the continuity of the map $(\varepsilon, u) \longmapsto D_{u} \Phi(\varepsilon, u)$ is proved. Analogously, the Gateaux derivative with respect to the first argument of $\Phi$ at $(\varepsilon, u) \in \mathbb{R}^{+} \times W_{p}^{2}(\Omega)$ is

$$
D_{\varepsilon} \Phi(\varepsilon, u) \equiv\left(\begin{array}{l}
0 \\
-\eta(\cdot) g(u)+f_{\partial \Omega} \eta(x) g(u) d \mathcal{H}^{n-1} \\
0
\end{array}\right)
$$


which is proved to be continuous in the same way above. Thus, $\Phi$ is continuously Fréchet differentiable and by similar reasonings one can conclude that $\Phi$ is smooth in Fréchet sense.

Step 2. $D_{u} \Phi(0, \bar{u}): W_{p}^{2}(\Omega) \longrightarrow \mathfrak{U} \times \mathbb{R}$ is a linear homeomorphism.

In fact, by the Open-Mapping Theorem, it suffices to prove that the continuous map $D_{u} \Phi(0, \bar{u})$ is a bijection. Thus, if $v$ lives in the null-space of $D_{u} \Phi(0, \bar{u})$, it follows that $v$ solves

$$
\begin{cases}\Delta v=0 & \text { in } \Omega \\ \frac{\partial v}{\partial \nu}=0 & \text { on } \quad \partial \Omega \\ g^{\prime}(\bar{u}) f_{\partial \Omega} \eta(x) v d \mathcal{H}^{n-1}=0 . & \end{cases}
$$

The two first equations imply $v$ constant and thanks to $(\mathbf{H})$ and $g^{\prime}(\bar{u}) \neq 0$ we have $v \equiv 0$, proving the injectivity of $D_{u} \Phi(0, \bar{u})$.

Now, given $(\phi, \psi, c) \in \mathfrak{U} \times \mathbb{R}$, since $\int_{\Omega} \phi d x=\int_{\partial \Omega} \psi d \mathcal{H}^{n-1}$, the linear problem

$$
\begin{cases}\Delta h=\phi & \text { in } \quad \Omega \\ \frac{\partial h}{\partial \nu}=\psi & \text { on } \quad \partial \Omega\end{cases}
$$

is solvable in $W_{p}^{2}(\Omega), p>n$ (see [18]), and choosing a suitable solution $h \in$ $W_{p}^{2}(\Omega)$ verifying the condition

$$
\frac{g^{\prime}(\bar{u})}{\mathcal{H}^{n-1}(\partial \Omega)} \int_{\partial \Omega} \eta(x) h d \mathcal{H}^{n-1}=c,
$$

we prove the surjectivity of $D_{u} \Phi(0, \bar{u})$, and so its bijectivity. Therefore, the previous steps allow us to apply the Implicit Function Theorem (cf. [6]) and to get a neighborhood of $\bar{u}$ in $W_{p}^{2}(\Omega), p>n$, on which $\bar{u}$ is the only solution to (4) for all $\varepsilon>0$ sufficiently small. The lemma is proved.

We are ready to prove the main result of this section.

Theorem 3.1. Every equilibrium solution to (1) is constant if $\varepsilon>0$ is sufficiently small.

Proof. We argue by contradiction. Assume that there is a sequence $\left\{u_{\varepsilon_{i}}\right\}$ of nonconstant equilibrium solutions to (1) such that $\varepsilon_{i} \rightarrow 0$, as $i \rightarrow \infty$. Thus, since $-1 \leq u_{\varepsilon_{i}} \leq 1$ in $\Omega$ are classical solutions to (4), [14], we have

$$
\begin{aligned}
\int_{\Omega}\left|\nabla u_{\varepsilon_{i}}\right|^{2} d x & =\varepsilon_{i} \int_{\partial \Omega} \eta(x) g\left(u_{\varepsilon_{i}}\right) u_{\varepsilon_{i}} d \mathcal{H}^{n-1} \\
& \leq \varepsilon_{i}\|\eta\|_{L^{\infty}(\partial \Omega)}\|g\|_{L^{\infty}([-1,1])} \mathcal{H}^{n-1}(\partial \Omega) \stackrel{i \rightarrow \infty}{\longrightarrow} 0 .
\end{aligned}
$$

Then $\left\{u_{\varepsilon_{i}}\right\}$ is bounded in $H^{1}(\Omega)$ what guarantees the existence of $\tilde{u} \in H^{1}(\Omega)$ and of a subsequence, again denoted by $\left\{u_{\varepsilon_{i}}\right\}$, such that as $i \rightarrow \infty$

- $u_{\varepsilon_{i}} \rightarrow \tilde{u}$ in $H^{1}(\Omega)$,

- $u_{\varepsilon_{i}} \rightarrow \tilde{u}$ a.e. in $\Omega$ and $\partial \Omega$,

- $u_{\varepsilon_{i}} \rightarrow \tilde{u}$ in $L^{2}(\partial \Omega)$. 
Considering the norm $|u|:=|| \mid \nabla u\left\|_{L^{2}(\Omega)}+\right\| u \|_{L^{2}(\partial \Omega)}$ in $H^{1}(\Omega)$ (equivalent to the usual one), where the boundary values are understood in the trace sense, from the convergences above it follows that

$$
|\tilde{u}| \leq \liminf _{i \rightarrow \infty}\left|u_{\varepsilon_{i}}\right|=\lim _{i \rightarrow \infty}\left[\left\||| \nabla u_{\varepsilon_{i}}||_{L^{2}(\Omega)}+\right\| u_{\varepsilon_{i}} \|_{L^{2}(\partial \Omega)}\right]=\|\tilde{u}\|_{L^{2}(\partial \Omega)} .
$$

Thus, $\||\nabla \tilde{u}|\|_{L^{2}(\Omega)}=0$ and then we have $-1 \leq \tilde{u} \leq 1$, with $\tilde{u}$ constant over $\Omega$. Further, from $(\mathbf{H})$ and

$$
\int_{\partial \Omega} \eta(x) g\left(u_{\varepsilon_{i}}\right) d \mathcal{H}^{n-1}=0
$$

for all $i$, we have $g(\tilde{u})=0$, that is, $\tilde{u} \equiv-1, \tilde{u} \equiv 0$ or $\tilde{u} \equiv 1$.

Now, for $p>n$, from the a priori elliptic $L^{p}$-estimate in [4], Proposition (3.3) there is a constant $C>0$ such that

$$
\begin{aligned}
\left\|u_{\varepsilon_{i}}-\tilde{u}\right\|_{W_{p}^{1}(\Omega)} & \leq C\left[\left\|\Delta\left(u_{\varepsilon_{i}}-\tilde{u}\right)\right\|_{L^{p}(\Omega)}+\left\|\varepsilon_{i} \eta(\cdot) g\left(u_{\varepsilon_{i}}\right)+\left(u_{\varepsilon_{i}}-\tilde{u}\right)\right\|_{L^{p}(\partial \Omega)}\right] \\
& \leq C\left[\varepsilon_{i}\left\|\eta(\cdot) g\left(u_{\varepsilon_{i}}\right)\right\|_{L^{p}(\partial \Omega)}+\left\|u_{\varepsilon_{i}}-\tilde{u}\right\|_{L^{p}(\partial \Omega)} \stackrel{i \rightarrow \infty}{\longrightarrow} 0\right.
\end{aligned}
$$

and thus

$$
u_{\varepsilon_{i}} \stackrel{i \rightarrow \infty}{\longrightarrow} \tilde{u} \quad \text { in } \quad W_{p}^{1}(\Omega), \quad p>n .
$$

Using the standard elliptic $L^{p}$-estimate from [2], keeping the last notation about the constants independents of $i$ in next estimates, it follows that

$$
\begin{aligned}
\left\|u_{\varepsilon_{i}}-\tilde{u}\right\|_{W_{p}^{2}(\Omega)} \leq & C\left[\left\|\varepsilon_{i} \eta(x) g\left(u_{\varepsilon_{i}}\right)\right\|_{W_{p}^{1-1 / p}(\partial \Omega)}+\left\|u_{\varepsilon_{i}}-\tilde{u}\right\|_{L^{p}(\Omega)}\right] \\
\leq & C\left[\varepsilon_{i}\left\|g\left(u_{\varepsilon_{i}}\right)\right\|_{W_{p}^{1}(\Omega)}+\left\|u_{\varepsilon_{i}}-\tilde{u}\right\|_{L^{p}(\Omega)}\right] \\
\leq & C\left[\varepsilon_{i}\left\|u_{\varepsilon_{i}}-\tilde{u}\right\|_{W_{p}^{1}(\Omega)}+\varepsilon_{i}\left\|g\left(u_{\varepsilon_{i}}\right)\right\|_{L^{p}(\Omega)}\right. \\
& \left.+\left\|u_{\varepsilon_{i}}-\tilde{u}\right\|_{L^{p}(\Omega)}\right] \stackrel{i \rightarrow \infty}{\longrightarrow} 0 .
\end{aligned}
$$

Then

$$
u_{\varepsilon_{i}} \stackrel{i \rightarrow \infty}{\longrightarrow} \tilde{u} \quad \text { in } \quad W_{p}^{2}(\Omega), \quad p>n
$$

and by Lemma 3.1 we get $u_{\varepsilon_{i}} \equiv \tilde{u}$ for all $i$ large enough, a contradiction.

Remark 3.1. Note that the results of this section, about nonexistence of nonconstant equilibria to (1), demanded only nonzero average of $\eta(\cdot)$ in $(\mathbf{H})$, that is, the nonexistence proved for $\varepsilon>0$ small holds true with no sign condition on $\eta(\cdot)$. However, in order to get existence results in the invariant sets $\mathfrak{M}^{-}$ and $\mathfrak{M}^{+}$, the requirement $\eta(\cdot)$ changes sign in $\partial \Omega$ is a necessary condition. Actually, as a consequence of divergence theorem, it is not possible to get nonconstant equilibria to (1) belonging to $\mathfrak{M}^{-}$or $\mathfrak{M}^{+}$for $\eta(\cdot)$ having a definite sign. 


\section{Existence of nonconstant equilibria}

Our aim now is to prove the existence of at least two nonconstant equilibria to (1). Using the variational method we will search for that kind of solutions in the invariant sets $\mathfrak{M}^{-}$and $\mathfrak{M}^{+}$introduced in Sect. 2. Note that this objective is attainable only if the parameter is not small, according to Theorem 3.1.

In the proof of the result of this section we will consider two cases related to the sign of the average over $\partial \Omega$ of the weight function $\eta(\cdot)$. In fact, the lower bound for the parameter produced by our approach determining existence of two nonconstant equilibria to (1) depends on the sign of $\int_{\partial \Omega} \eta(x) d \mathcal{H}^{n-1}$. The result is so established by getting minimizers in the closed convex sets $\mathfrak{M}^{-}$ and $\mathfrak{M}^{+}$of $H^{1}(\Omega)$, which are proved to be nonconstant weak solutions to (4) for $\varepsilon>0$ large enough.

Let us first state a lemma needed later.

Lemma 4.1. Let $\omega \in C^{1, \gamma}(\partial \Omega)$, where $0<\gamma<1$, be a function which changes sign on $\partial \Omega$. The number

$$
\varepsilon_{0}=\inf \left\{\frac{\int_{\Omega}|\nabla v|^{2} d x}{\int_{\partial \Omega} \omega(x) v^{2} d \mathcal{H}^{n-1}}: v \in H^{1}(\Omega) \text { and } \int_{\partial \Omega} \omega(x) v^{2} d \mathcal{H}^{n-1}>0\right\}
$$

is positive if $\omega(\cdot)$ has a negative average over $\partial \Omega$, and equals to zero otherwise. Further, the problem

$$
\begin{cases}\Delta v=0 & \text { in } \quad \Omega \\ \frac{\partial v}{\partial \nu}=\varepsilon_{0} \omega(x) v & \text { on } \quad \partial \Omega,\end{cases}
$$

has a positive solution.

Proof. For a proof of this result, see [17].

The result to be proved in this section is

Theorem 4.1. Problem (1) has at least two nonconstant equilibrium solutions for all $\varepsilon>0$ sufficiently large.

Proof. From the symmetry of $g$ it follows that $u \in \mathfrak{M}^{+}$is a nonconstant equilibrium solution to (1) if, and only if, $-u \in \mathfrak{M}^{-}$is another one. Thus, it suffices to prove the existence of a nonconstant equilibrium solution to (1) belonging to $\mathfrak{M}^{+}$.

Using the direct method of the Calculus of Variations one can prove that $\left.\mathcal{E}_{\varepsilon}\right|_{\mathfrak{M}^{+}}$is bounded from below and there exists $u_{\varepsilon} \in \mathfrak{M}^{+}$such that

$$
\mathcal{E}_{\varepsilon}\left(u_{\varepsilon}\right)=\inf _{v \in \mathfrak{M}^{+}} \mathcal{E}_{\varepsilon}(v), \quad \forall \varepsilon>0
$$

We will split the rest of the proof in two steps.

Step 1. $u_{\varepsilon}$ is a weak solution to (4) for all $\varepsilon>0$. 
The argument in this step is inspired [16] where a homogeneous Dirichlet boundary value problem was considered. We shall prove that $\mathcal{E}_{\varepsilon}$ has zero derivative in all directions in $H^{1}(\Omega)$, that is

$$
D \mathcal{E}_{\varepsilon}\left(u_{\varepsilon}\right) \phi:=\int_{\Omega} \nabla u_{\varepsilon} \cdot \nabla \phi d x-\varepsilon \int_{\partial \Omega} \eta(x) g\left(u_{\varepsilon}\right) \phi d \mathcal{H}^{n-1}=0, \quad \forall \phi \in H^{1}(\Omega) .
$$

By density, it suffices to prove the last equality for any $\phi \in C^{\infty}(\bar{\Omega})$. Fix $\tau>0$ and define

$$
v_{\tau}:=\min \left\{1, \max \left\{0, u_{\varepsilon}+\tau \phi\right\}\right\} .
$$

It is not difficult to see that $v_{\tau} \in \mathfrak{M}^{+}$and that $v_{\tau}=u_{\varepsilon}+\tau \phi-\phi^{\tau}+\phi_{\tau}$, where

$$
\phi^{\tau}:=\max \left\{0, u_{\varepsilon}+\tau \phi-1\right\} \quad \text { and } \quad \phi_{\tau}:=-\min \left\{0, u_{\varepsilon}+\tau \phi\right\}
$$

are such that $\phi^{\tau}, \phi_{\tau} \geq 0$ and $\phi^{\tau}, \phi_{\tau} \in H^{1}(\Omega)$. Note that for $t$ small $u_{\varepsilon}+t\left[v_{\tau}-\right.$ $\left.u_{\varepsilon}\right]=t v_{\tau}+(1-t) u_{\varepsilon} \in \mathfrak{M}^{+}$given that $\mathfrak{M}^{+}$is convex. Since $\mathcal{E}_{\varepsilon}$ is differentiable in the direction $v_{\tau}-u_{\varepsilon}$ and $u_{\varepsilon}$ minimizes $\left.\mathcal{E}_{\varepsilon}\right|_{\mathfrak{M}^{+}}$we have

$$
0 \leq D \mathcal{E}_{\varepsilon}\left(u_{\varepsilon}\right)\left(v_{\tau}-u_{\varepsilon}\right)=\tau D \mathcal{E}_{\varepsilon}\left(u_{\varepsilon}\right) \phi-D \mathcal{E}_{\varepsilon}\left(u_{\varepsilon}\right) \phi^{\tau}+D \mathcal{E}_{\varepsilon}\left(u_{\varepsilon}\right) \phi_{\tau}
$$

and thus

$$
D \mathcal{E}_{\varepsilon}\left(u_{\varepsilon}\right) \phi \geq \frac{1}{\tau}\left[D \mathcal{E}_{\varepsilon}\left(u_{\varepsilon}\right) \phi^{\tau}-D \mathcal{E}_{\varepsilon}\left(u_{\varepsilon}\right) \phi_{\tau}\right] .
$$

Defining the sets

$$
\Omega^{\tau} \doteq\left\{x \in \Omega: u_{\varepsilon}(x)+\tau \phi(x) \geq 1>u_{\varepsilon}(x)\right\}
$$

and

$$
\partial \Omega^{\tau} \doteq\left\{x \in \partial \Omega: u_{\varepsilon}(x)+\tau \phi(x) \geq 1>u_{\varepsilon}(x)\right\},
$$

where the functions in $\partial \Omega^{\tau}$ are understood in the trace sense, and denoting

$$
\{\eta \geq 0\}=\{x \in \partial \Omega: \eta(x) \geq 0\} \quad \text { and } \quad\{\eta<0\}=\{x \in \partial \Omega: \eta(x)<0\}
$$

it follows that

$$
\begin{aligned}
D \mathcal{E}_{\varepsilon}\left(u_{\varepsilon}\right) \phi^{\tau} & =\int_{\Omega^{\tau}} \nabla u_{\varepsilon} \cdot \nabla\left[u_{\varepsilon}+\tau \phi-1\right] d x-\varepsilon \int_{\partial \Omega^{\tau}} \eta(x) g\left(u_{\varepsilon}\right)\left[u_{\varepsilon}+\tau \phi-1\right] d \mathcal{H}^{n-1} \\
& \geq \tau \int_{\Omega^{\tau}} \nabla u_{\varepsilon} \cdot \nabla \phi d x-\varepsilon \int_{\partial \Omega^{\tau} \cap\{\eta \geq 0\}} \eta(x) g\left(u_{\varepsilon}\right)\left[u_{\varepsilon}+\tau \phi-1\right] d \mathcal{H}^{n-1} \\
& \geq \tau \int_{\Omega^{\tau}} \nabla u_{\varepsilon} \cdot \nabla \phi d x-\varepsilon \tau \int_{\partial \Omega^{\tau} \cap\{\eta \geq 0\}} \eta(x) g\left(u_{\varepsilon}\right) \phi d \mathcal{H}^{n-1} .
\end{aligned}
$$

Since $\mathcal{L}^{n}\left(\Omega^{\tau}\right)$ and $\mathcal{H}^{n-1}\left(\partial \Omega^{\tau}\right)$, the Lebesgue and Haursdorff measures, respectively, go to zero as $\tau \rightarrow 0$, the absolute continuity of Lebesgue type integrals implies

$$
D \mathcal{E}_{\varepsilon}\left(u_{\varepsilon}\right) \phi^{\tau} \geq o(\tau)
$$

as $\tau \rightarrow 0$. In a similar way we can get

$$
D \mathcal{E}_{\varepsilon}\left(u_{\varepsilon}\right) \phi_{\tau} \leq o(\tau)
$$


as $\tau \rightarrow 0$ and then, by (7),

$$
D \mathcal{E}_{\varepsilon}\left(u_{\varepsilon}\right) \phi \geq 0
$$

The procedure above was independent of the sign of $\phi$ and so, working with $-\phi$, we are able to conclude that the last inequality also holds with reversed sign. Therefore,

$$
D \mathcal{E}_{\varepsilon}\left(u_{\varepsilon}\right) \phi=0, \quad \forall \phi \in C^{\infty}(\bar{\Omega}),
$$

and $u_{\varepsilon}$ is a weak solution to (4) for all $\varepsilon>0$.

Step 2. $u_{\varepsilon}$ is nonconstant for all $\varepsilon>0$ sufficiently large.

We will consider two cases.

Case 1. $\int_{\partial \Omega} \eta(x) d \mathcal{H}^{n-1}<0$.

Consider $\varepsilon_{0}$ given by $(5)$ with $\omega(\cdot)=\eta(\cdot)$. In this case, by Lemma 4.1 , $\varepsilon_{0}>0$ and problem (6) has a positive solution $\xi$, which is a classical one. Moreover, by Green's identity and the maximum principle, $\xi>0$ in $\bar{\Omega}$ is nonconstant. Choose $a>0$ small in such way that $a \xi \in \mathfrak{M}^{+}$. Multiplying the first equation of (6) by $\xi$ and integrating by parts it follows that

$$
\int_{\Omega}|\nabla \xi|^{2} d x=\varepsilon_{0} \int_{\partial \Omega} \eta(x) \xi^{2} d \mathcal{H}^{n-1}
$$

and the same procedure yields

$$
\int_{\Omega} \xi^{2}|\nabla \xi|^{2} d x=\frac{\varepsilon_{0}}{3} \int_{\partial \Omega} \eta(x) \xi^{4} d \mathcal{H}^{n-1} .
$$

Thus, we have

$$
\begin{aligned}
\mathcal{E}_{\varepsilon}(a \xi) & =\frac{a^{2}}{2} \int_{\Omega}|\nabla \xi|^{2} d x-\varepsilon \int_{\partial \Omega} \eta(x)\left[\frac{a^{2} \xi^{2}}{2}-\frac{a^{4} \xi^{4}}{4}\right] d \mathcal{H}^{n-1} \\
& =\frac{a^{2}\left(\varepsilon_{0}-\varepsilon\right)}{2} \int_{\partial \Omega} \eta(x) \xi^{2} d \mathcal{H}^{n-1}+\frac{\varepsilon a^{4}}{4} \int_{\partial \Omega} \eta(x) \xi^{4} d \mathcal{H}^{n-1} \\
& =\frac{a^{2}\left(\varepsilon_{0}-\varepsilon\right)}{2 \varepsilon_{0}} \int_{\Omega}|\nabla \xi|^{2} d x+\frac{3 \varepsilon a^{4}}{4 \varepsilon_{0}} \int_{\Omega} \xi^{2}|\nabla \xi|^{2} d x \\
& =\frac{a^{2}}{2 \varepsilon_{0}}\left[\left(\varepsilon_{0}-\varepsilon\right) \int_{\Omega}|\nabla \xi|^{2} d x+\frac{3 \varepsilon a^{2}}{2} \int_{\Omega} \xi^{2}|\nabla \xi|^{2} d x\right] .
\end{aligned}
$$

Therefore, for each $\varepsilon>\varepsilon_{0}$ we have

$$
\mathcal{E}_{\varepsilon}(a \xi) \longrightarrow 0^{-} \quad \text { as } \quad a \rightarrow 0^{+}
$$

and then

$$
\mathcal{E}_{\varepsilon}\left(u_{\varepsilon}\right)<0, \quad \forall \varepsilon>\varepsilon_{0} .
$$

Once the constant equilibrium solutions to (1) satisfy

$$
\mathcal{E}_{\varepsilon}(-1)>0, \quad \mathcal{E}_{\varepsilon}(0)=0 \quad \text { and } \quad \mathcal{E}_{\varepsilon}(1)>0,
$$

it follows that $u_{\varepsilon}$ is nonconstant.

Case 2. $\int_{\partial \Omega} \eta(x) d \mathcal{H}^{n-1}>0$. 
This case can be treated similarly to the first one, considering now $\varepsilon_{0}$ given by $(5)$ with $\omega(\cdot)=-2 \eta(\cdot)$ and noting that in this way $\int_{\partial \Omega} \omega(x) d \mathcal{H}^{n-1}<$ 0 .

Remark 4.1. The proof of Theorem 4.1 yields a lower bound for the parameter determining existence of nontrivial equilibria. Namely, problem (1) has at least two nontrivial equilibria for all $\varepsilon>\varepsilon_{0}$, where $\varepsilon_{0}$ is given by (5) with $\omega(\cdot)=\eta(\cdot)$ if the average of $\eta(\cdot)$ over $\partial \Omega$ is negative, and $\omega(\cdot)=-2 \eta(\cdot)$ if $\eta(\cdot)$ has a positive average over $\partial \Omega$.

Remark 4.2. It should be noted that the argument in Step 1 of the proof of Theorem 4.1 in proving that $u_{\varepsilon}$ is a weak solution to (4) not demanded that $\mathcal{E}_{\varepsilon}$ was a $C^{1}$-functional. So it can be applied to a more general class of problems.

Remark 4.3. If one knew that the nonconstant equilibria $u_{\varepsilon}$ and $-u_{\varepsilon}$ given by Theorem 4.1 were isolated (we will see in next section this actually occurs) since (1) generates a nonlinear dynamical system in $H^{1}(\Omega)$, having $\mathcal{E}_{\varepsilon}$ as a Lyapunov function, one would have $u_{\varepsilon} \in \mathfrak{M}^{+}$and $-u_{\varepsilon} \in \mathfrak{M}^{-}$asymptotically stable nonconstant equilibria to (1) for all $\varepsilon>\varepsilon_{0}$. But we will be able to prove even more, namely, the solutions of the parabolic problem (1) beginning next to $u_{\varepsilon}$ and $-u_{\varepsilon}$ decay at a exponential rate to it, for $\varepsilon>\varepsilon_{0}$.

\section{Exponential stability of nonconstant equilibria}

This section is devoted to proving that the nonconstant equilibria $-u_{\varepsilon} \in \mathfrak{M}^{-}$ and $u_{\varepsilon} \in \mathfrak{M}^{+}$to (1) given by Theorem 4.1 are exponentially stable, besides concluding they are isolated and depend smoothly on the parameter $\varepsilon$.

It is known that there is a linearized stability principle for problem (1) (see $[10,13]$ ), which is based on analysing the spectrum of the eigenvalue problem

$$
\begin{cases}\Delta \varphi=\mu \varphi & \text { in } \quad \Omega \\ \frac{\partial \varphi}{\partial \nu}=\varepsilon \eta(x)\left(1-3 u_{\varepsilon}^{2}\right) \varphi & \text { on } \quad \partial \Omega .\end{cases}
$$

Since the functional $\mathcal{E}_{\varepsilon}$ given by $(3)$ is smooth if $g$ satisfies appropriate growth conditions at infinity (what can be assumed since $\pm u_{\varepsilon}$ are bounded uniformly on $\varepsilon$ ), the basic problem is to localize the first eigenvalue of (8), given by

$$
\mu_{1}(\varepsilon)=\sup _{v \in H^{1}(\Omega) \backslash\{0\}}\left\{\frac{-\mathcal{E}_{\varepsilon}^{\prime \prime}\left(u_{\varepsilon}\right)(v, v)}{\|v\|_{L^{2}(\Omega)}^{2}}\right\} .
$$

In fact, to infer about stability of $\pm u_{\varepsilon}$ using the linearized stability principle we need to check if $\mu_{1}(\varepsilon) \neq 0$. Actually, $\mu_{1}(\varepsilon)=0$ is the critical case of stability for which the principle does not apply. Thus, next result will be crucial to prove that the above mentioned solutions are exponentially stable, and will be also an important ingredient for applying the Implicit Function Theorem in order to get isolation and smooth dependence on the parameter. 
Lemma 5.1. Zero is not an eigenvalue to (8) for all $\varepsilon>\varepsilon_{0}$.

Proof. By contradiction, suppose the contrary holds true. Then, there exists a solution $\varphi \not \equiv 0$ to (8), which is a classical one. Once $0<u_{\varepsilon}<1$ in $\bar{\Omega}$ by the maximum principle and Hopf's Lemma, the function $\Upsilon: \bar{\Omega} \longrightarrow \mathbb{R}$ given by

$$
\Upsilon(x):=\frac{\varphi(x)}{u_{\varepsilon}(x)\left(1-u_{\varepsilon}^{2}(x)\right)}
$$

is well-defined and smooth. Direct computations show that $\Upsilon$ is a solution to the second order elliptic boundary value problem

$$
\begin{cases}\Delta \Upsilon+\sum_{j=1}^{n} b_{j}(x) \frac{\partial \Upsilon}{\partial x_{j}}+c(x) \Upsilon=0 & \text { in } \Omega \\ \frac{\partial \Upsilon}{\partial \nu}=0 & \text { on } \partial \Omega\end{cases}
$$

where $b_{j} \in C^{1, \theta}(\bar{\Omega})$ and $c(\cdot)=-\beta(\cdot)\left|\nabla u_{\varepsilon}\right|^{2}$, with $\beta \in C^{1, \theta}(\bar{\Omega})$ being a nonnegative function and $0<\theta<1$.

Now, since $c(\cdot) \leq 0$ in $\Omega$, the maximum principle and Hopf's Lemma imply that the maximum of $\Upsilon$ occurs at a point of $\partial \Omega$ on which $\partial \Upsilon / \partial \nu>0$, if $\Upsilon$ was not a constant. Thus, $\Upsilon$ must be constant, in fact equals to zero by (10). Therefore, by the definition of $\Upsilon$ we may infer that $\varphi \equiv 0$ in $\Omega$, a contradiction.

We are in position now to precisely locate the first eigenvalue of problem (8) and to prove one of the central results of this paper.

Theorem 5.1. Problem (1) admits at least two nonconstant exponentially stable equilibria for all $\varepsilon>0$ sufficiently large.

Proof. Since the nonconstant equilibria $-u_{\varepsilon}$ and $u_{\varepsilon}$ to (1) given by Theorem 4.1 for each $\varepsilon>\varepsilon_{0}$ are such that

$$
-1<-u_{\varepsilon}<0 \text { and } 0<u_{\varepsilon}<1 \text { in } \bar{\Omega}
$$

by the maximum principle, using the embedding $W_{p}^{1}(\Omega) \hookrightarrow C(\bar{\Omega})$ for $p>n$, [1], it is not difficult to see that $-u_{\varepsilon}$ and $u_{\varepsilon}$ are interior points of the sets

$$
\widetilde{\mathfrak{M}}^{-} \doteq \mathfrak{M}^{-} \cap W_{p}^{1}(\Omega) \quad \text { and } \quad \widetilde{\mathfrak{M}}^{+} \doteq \mathfrak{M}^{+} \cap W_{p}^{1}(\Omega)
$$

respectively, in the $W_{p}^{1}(\Omega)$-topology $(p>n)$. Also, recalling Theorem 4.1 one can verify that

$$
\inf _{v \in \widetilde{\mathfrak{M}}^{-}} \mathcal{E}_{\varepsilon}(v)=\mathcal{E}_{\varepsilon}\left(-u_{\varepsilon}\right)
$$

and

$$
\inf _{v \in \widetilde{\mathfrak{M}}^{+}} \mathcal{E}_{\varepsilon}(v)=\mathcal{E}_{\varepsilon}\left(u_{\varepsilon}\right)
$$

that is, $-u_{\varepsilon}$ and $u_{\varepsilon}$ are local minima, in the $W_{p}^{1}(\Omega)$-topology, of $\mathcal{E}_{\varepsilon}$ acting in $W_{p}^{1}(\Omega), p>n$. Thus, we have

$$
\mathcal{E}_{\varepsilon}^{\prime \prime}\left(u_{\varepsilon}\right)(v, v) \geq 0 \quad \text { and } \quad \mathcal{E}_{\varepsilon}^{\prime \prime}\left(-u_{\varepsilon}\right)(v, v) \geq 0
$$


for all $v \in W_{p}^{1}(\Omega)$ and so, by density, $\mu_{1}(\varepsilon)$ given by (9) is such that $\mu_{1}(\varepsilon) \leq 0$. But Lemma 5.1 implies $\mu_{1}(\varepsilon)<0$ and then the spectrum of $(8)$ lies in

$$
\left\{z \in \mathbb{C}: \operatorname{Re} z<\mu_{1}(\varepsilon)\right\}
$$

for all $\varepsilon>\varepsilon_{0}$. Therefore, the result follows from the linearized stability principle.

As a consequence of Lemma 5.1 and the Implicit Function Theorem we can prove isolation and smooth dependence on the parameter for nonconstant equilibrium solutions in next

Theorem 5.2. The nonconstant equilibrium solutions $u_{\varepsilon} \in \mathfrak{M}^{+},-u_{\varepsilon} \in \mathfrak{M}^{-}$to (1) are isolated for all $\varepsilon>\varepsilon_{0}$. Moreover, the maps

$$
\left(\varepsilon_{0},+\infty\right) \ni \varepsilon \longmapsto u_{\varepsilon} \in \mathfrak{M}^{+},
$$

and

$$
\left(\varepsilon_{0},+\infty\right) \ni \varepsilon \longmapsto-u_{\varepsilon} \in \mathfrak{M}^{-}
$$

are smooth.

Proof. Let us argue for $\mathfrak{M}^{+}$since the other case is similar. First of all, it follows from Theorem 4.1 that the maps above are well defined. Now, introduce the smooth map $\mathscr{G}: \mathbb{R}^{+} \times W_{p}^{2}(\Omega) \longrightarrow L^{p}(\Omega) \times W_{p}^{1-1 / p}(\partial \Omega), p>n$, given by

$$
\mathscr{G}(\varepsilon, u)=\left(\Delta u, \frac{\partial u}{\partial \nu}-\varepsilon \eta(\cdot) u\left(1-u^{2}\right)\right) .
$$

Note that

$$
\mathscr{G}\left(\varepsilon, u_{\varepsilon}\right)=0=\mathscr{G}\left(\varepsilon,-u_{\varepsilon}\right), \quad \forall \varepsilon>\varepsilon_{0} .
$$

Further, the operator $D_{u} \mathscr{G}\left(\varepsilon, u_{\varepsilon}\right): W_{p}^{2}(\Omega) \longrightarrow L^{p}(\Omega) \times W_{p}^{1-1 / p}(\partial \Omega)$ given by

$$
D_{u} \mathscr{G}\left(\varepsilon, u_{\varepsilon}\right) \cdot v=\left(\Delta v, \frac{\partial v}{\partial \nu}-\varepsilon \eta(\cdot)\left(1-3 u_{\varepsilon}^{2}\right) v\right)
$$

is injective by Lemma 5.1 and surjective since it is a Fredholm operator of zero index [12], and thus a bijection. Thus, the Implicit Function Theorem provides a neighborhood of $u_{\varepsilon}$ in $\mathbb{R}^{+} \times W_{p}^{2}(\Omega)(p>n)$ such that all solutions to (4) near $u_{\varepsilon}$ lie within it. Also from the beginning of the proof of Theorem 5.1 we have that $u_{\varepsilon}$ is an interior point of $\mathfrak{M}^{+}$in the topology of $W_{p}^{1}(\Omega)(p>n)$ and $0<u_{\varepsilon}<1$ in $\bar{\Omega}$ for $\varepsilon>\varepsilon_{0}$. Since the embedding $W_{p}^{1}(\Omega) \hookrightarrow C(\bar{\Omega})$ for $p>n$ holds true [1], reducing the mentioned neighborhood, if necessary, all the solutions for varying $\varepsilon$ to (4) near $u_{\varepsilon}$ lie in $\mathfrak{M}^{+}$.

Moreover, the Implicit Function Theorem yields the desired smooth dependence on the parameter $\varepsilon$, proving the theorem. 


\section{References}

[1] Adams, R.A., Fournier, J.J.F.: "Sobolev Spaces", Second edn. Elsevier, Netherlands (2003)

[2] Agmon, S., Douglis, A., Nirenberg, L.: Estimates near the boundary for solutions of elliptic partial differential equations satisfying general boundary conditions, I. Commun. Pure Appl. Math. 12, 623-727 (1959)

[3] Alikakos, N.D.: Regularity and asymptotic behavior for the second order parabolic equation with nonlinear boundary conditions in $L^{p}$. J. Diff. Eqns. 39, 311$344(1981)$

[4] Amann, H.: Nonlinear elliptic equations with nonlinear boundary conditions. In: Eckhaus, W. (ed.) "New Developments in Differential Equations", pp. 43-63. North-Holland, Amsterdam (1976)

[5] Amann, H.: Parabolic evolution equations and nonlinear boundary conditions. J. Diff. Eqns. 72, 201-269 (1988)

[6] Ambrosetti, A., Prodi, G.: A Primer of Nonlinear Analysis. Cambridge University Press, Cambridge (1993)

[7] Arrieta, J.M., Carvalho, A.N., Rodríguez-Bernal, A.: Attractors of parabolic problems with nonlinear boundary conditions. Uniform bounds. Commun. Part. Diff. Eqns. 25, 1-37 (2000)

[8] Arrieta, J.M., Cònsul, N., Rodríguez-Bernal, A.: Stable boundary layers in a diffusion problem with nonlinear reaction at the boundary. Z. Angew. Math. Phys. 55, 1-14 (2004)

[9] Ball, J.M., Taheri, A., Winter, M.: Local minimizers in micromagnetics and related problems. Calc. Var. 14, 1-27 (2002)

[10] Cònsul, N.: On equilibrium solutions of diffusion equations with nonlinear boundary conditions. Z. Angew Math. Phys. 47, 194-209 (1996)

[11] Cònsul, N., Solà-Morales, J.: Stability of local minima and stable nonconstant equilibria. J. Diff. Eqns. 157, 61-81 (1999)

[12] Grisvard, P.: Elliptic Problems in Nonsmooth Domains. Pitman, London (1985)

[13] Henry, D.: "Geometric theory of semilinear parabolic equations. In: Lecture Notes in Mathamatics, vol. 840. Springer, Berlin (1989)

[14] Madeira, G.F.: Existence and regularity for a nonlinear boundary flow problem of population genetics. Nonlinear Anal. 70, 974-981 (2009)

[15] Pao, C.V.: Nonlinear Parabolic and Elliptic Equations. Plenum Press, New York (1992)

[16] Struwe, M.: Variational Methods. Third edn. Springer, Berlin (2000) 
[17] Umezu, K.: On eigenvalue problems with Robin type boundary conditions having indefinite coefficients. Appl. Anal. 85, 1313-1325 (2006)

[18] Valent, T.: Boundary Value Problems of Finite Elasticity. Springer, New York (1988)

G. F. Madeira, A. S. do Nascimento

Departamento de Matemática,

Universidade Federal de São Carlos

13.565-905, São Carlos (SP),

Brazil

e-mail: gfmadeira@dm.ufscar.br

e-mail: arnaldon@dm.ufscar.br

Received: 23 April 2010.

Accepted: 18 February 2011. 